Rios LT, Aguiar VNP, Machado FC, Rocha CT, Neves BG. Bruxismo infantil e sua associação com fatores psicológicos - revisão sistemática da literatura. Rev. Odontol. Univ. Cid. São Paulo 2018 jan-mar; 30(1): 64-76

ISSN 1983-5183

\title{
BRUXISMO INFANTIL E SUA ASSOCIAÇÃO COM FATORES PSICOLÓGICOS - REVISÃO SISTEMÁTICA DA LITERATURA
}

\author{
Lisandra Teixeira RIOS 1 \\ lisandratrios@gmail.com \\ Valdelya Nara Pereira AGUIAR ${ }^{2}$ \\ valdelya.aguiar@gmail.com \\ Fernanda Campos MACHADO ${ }^{3}$ \\ fercampo@terra.com.br \\ Cristiane Tomaz ROCHA ${ }^{4}$ \\ cristianetomaz@hotmail.com \\ Beatriz Gonçalves NEVES 5 \\ beatrizgneves@gmail.com
}

\section{RESUMO}

O bruxismo compreende uma atividade parafuncional caracterizado pelo ato de ranger ou apertar os dentes de forma voluntária e/ou involuntariamente, ocorrendo durante o período diurno e/ou noturno, podendo acarretar vários prejuízos ao sistema estomatognático. Diversos são os fatores etiológicos que podem estar associados a esta desordem, como fatores locais, sistêmicos, hereditários, psicossociais e comportamentais. Pesquisadores têm sugerido que fatores comportamentais, como estresse, ansiedade e traços de personalidade se sobressaem a fatores locais. Portanto, o objetivo deste estudo foi realizar uma revisão sistemática da literatura a fim de avaliar se há relação entre bruxismo e fatores psicológicos em crianças. Foram realizados levantamentos nas bases de dados: PubMed, BVS Biblioteca Virtual em Saúde e SciELO no período de fevereiro de 2007 a setembro de 2017. Os descritores utilizados foram "bruxism", "children", "stress", "anxiety", "pshychological factors". Sendo assim, 97 publicações foram identificadas na busca inicial. Após aplicação dos critérios de inclusão e exclusão, a amostra final foi composta por sete estudos. Houve evidência significativa de uma associação entre estresse, ansiedade e fatores psicológicos com o bruxismo infantil. Porém, ainda existe uma enorme necessidade de estudos bem delineados e com metodologias padronizadas a fim de verificar a associação entre o bruxismo e fatores psicológicos.

PALAVRAS-CHAVE: BRUXISMO. CRIANÇA. ESTRESSE PSICOLÓGICO. ANSIEDADE.

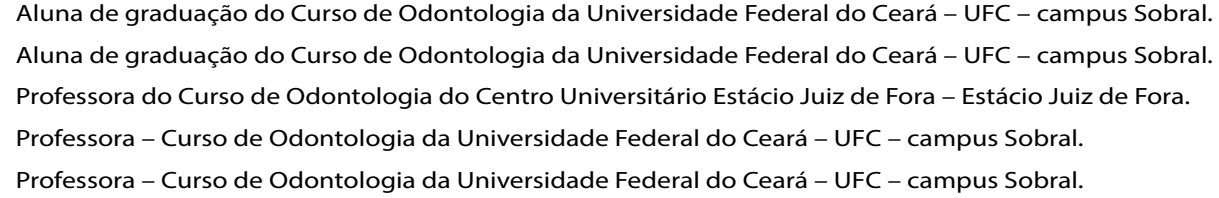


Rios LT, Aguiar VNP, Machado FC, Rocha CT, Neves BG. Bruxismo infantil e sua associação com fatores psicológicos - revisão sistemática da

literatura. Rev. Odontol. Univ. Cid. São Paulo 2018 jan-mar; 30(1): 64-76

ISSN 1983-5183

\title{
BRUXISM IN CHILDREN AND ITS ASSOCIATION WITH PSYCHOLOGICAL FACTORS - A SYSTEMATIC REVIEW OF LITERATURE
}

\begin{abstract}
Bruxism comprises a parafunctional activity characterized by the act of grinding or clenching the teeth voluntarily and/or involuntarily, occurring during the day and / or night, and may cause several damages to the stomatognathic system. Several etiological factors may be associated with this disorder, such as local, systemic, hereditary, psychosocial and behavioral factors. Researchers have suggested that behavioral factors such as stress, anxiety, and personality traits stand out from local factors. Therefore, the aim of this study was to perform a systematic review of the literature in order to evaluate if there is a relation between bruxism and psychological factors in children. Data were collected in the databases: PubMed, BVS Virtual Health Library and SciELO from February 2007 to September 2017. The descriptors used were "bruxism", "children", "stress", "anxiety" and "pshychological factors". Thus, 97 publications were identified in the initial search. After applying the inclusion and exclusion criteria, the final sample consisted of seven studies. There was significant evidence of an association between stress, anxiety, and psychological factors with infant bruxism. However, there is still a great need for well-designed studies with standardized methodologies in order to verify the association between bruxism and psychological factors.
\end{abstract}

KEY WORDS: BRUXISM. CHILD. STRESS, PSYCHOLOGICAL. ANXIETY.

\section{INTRODUÇÃO}

O bruxismo é caracterizado pela atividade noturna e/ou diurna involuntária dos músculos mastigatórios, rítmica ou espasmódica, podendo apresentar apertamento e/ou ranger dos dentes ${ }^{1}$. Neste último caso, ocorrem contrações rítmicas musculares, sendo mais frequentes durante o sono. Durante essa atividade parafuncional, que acontece quase inteiramente em nível subconsciente, os mecanismos de proteção neuromuscular estão ausentes, podendo acarretar danos ao sistema mastigatório e desordens temporomandibulares ${ }^{2}$, além de dores musculares e de cabeça, desgastes dentários, prejuízo aos tecidos periodontais, problemas respiratórios, distúrbios do sono e prejuízos na fala ${ }^{3,4}$.

Há uma considerável discrepância na literatura em relação à prevalência de bruxismo. Estudos epidemiológicos com diferentes metodologias e populações têm sido realizados a fim de verificar esse dado ${ }^{5}$. De acordo com Manfredini et al. ${ }^{6}$ (2004), observou-se uma grande variabilidade na prevalência de bruxismo em crianças entre os diferentes estudos avaliados (3,5\% e 40,6\%), ocorrendo uma diminuição com a idade e sem diferenças em relação ao gênero. No Brasil, em um estudo avaliando bruxismo em escolares, o dado de prevalência foi de $43 \% 7$. Em uma revisão sistemática avaliando a prevalência de bruxismo do sono na infância, verificou-se que as taxas de prevalência variaram de 5,9\% a 49,6\%, sendo que essas variações apresentaram possíveis associações com os critérios diagnósticos para bruxismo ${ }^{8}$.

A etiologia dessa atividade parafuncional é complexa e controversa ${ }^{9}$, estando relacionada a fatores locais, sistêmicos, ocupacionais, hereditários e psicológicos ${ }^{10}$. Pesquisadores têm sugerido que fatores comportamentais, como estresse, ansiedade e características de personalidade se sobressaem a fatores locais ${ }^{8}$, sendo o estresse emocional o fator etiológico mais fortemente associado a essa parafunção nas últimas décadas ${ }^{11}$. Em estudo recente, a ansiedade foi apontada como o principal fator que interferiu na qualidade de vida de crianças brasileiras com bruxismo noturno ${ }^{12}$. 
Rios LT, Aguiar VNP, Machado FC, Rocha CT, Neves BG. Bruxismo infantil e sua associação com fatores psicológicos - revisão sistemática da literatura. Rev. Odontol. Univ. Cid. São Paulo 2018 jan-mar; 30(1): 64-76

ISSN 1983-5183

O diagnóstico do bruxismo ainda é um desafio para o cirurgião-dentista. Devido a sua natureza multifatorial, torna-se importante estabelecer o diagnóstico com base nos possíveis fatores etiológicos e não apenas nos sinais clínicos ${ }^{6}$. Um questionário investigando a história médica do paciente, presença de hábitos parafuncionais, alterações sistêmicas e neurológicas, estilo de vida e qualidade de vida, relações familiares e sociais do paciente, aliado a um exame abrangente de sinais e sintomas clínicos, constitui o protocolo de avaliação-padrão para o diagnóstico de bruxismo. Assim, a identificação de fatores psicossociais como ansiedade, estresse e características de personalidade deve ser considerada para um melhor entendimento no desenvolvimento do bruxismo ${ }^{13}$.

Considerando que a etiologia do bruxismo não está completamente esclarecida e uma vez que há controvérsias nas pesquisas se fatores psicológicos podem influenciar no desencadeamento e manutenção dessa desordem, o presente estudo tem como objetivo avaliar, através de uma revisão sistematizada da literatura, a relação entre o bruxismo e fatores psicológicos em crianças.

\section{METODOLOGIA}

Para esta revisão sistemática, foram incluídos todos os estudos relacionados ao bruxismo em crianças e fatores psicológicos, encontrados através das estratégias de busca definidas e que preencheram os critérios de elegibilidade. O método incluiu estratégia de busca, critérios de inclusão, critérios de exclusão, identificação dos estudos, extração e apresentação dos dados. Uma busca on-line foi realizada nas bases de dados PubMed, BVS Biblioteca Virtual em Saúde e SciELO no período de setembro de 2017. As palavraschave utilizadas para esta pesquisa foram "bruxism" "children", "anxiety", "stress" e "psychological factors". Nesta revisão, o descritor "psychological factors" foi usado como um termo genérico para agrupar todos os agentes psicológicos que pudessem apresentar um efeito no bruxismo em crianças, como, por exemplo, estresse, ansiedade e alterações de humor, traços temperamentais e emoções. A seguinte estratégia de busca foi aplicada: bruxism and children and anxiety; bruxism and children and stress e bruxism and children and psychological factors.

A amostra de estudos selecionada desta revisão obedeceu aos seguintes critérios de inclusão: (a) estudos com o objetivo de verificar a relação de fatores psicológicos com o bruxismo em criança; (b) indivíduos com idade de 0 a 12 anos foram considerados como crianças; (c) estudos com os seguintes critérios de diagnóstico para bruxismo: história, questionário ou entrevista com os pais, avaliação clínica ou polissonografia; (d) estudos publicados no período de fevereiro de 2007 a setembro de 2017 nas línguas portuguesa e inglesa, (e) estudos que tenham utilizado algum tipo de teste psicológico; (f) estudos randomizados, ensaios clínicos e casos-controle, que demonstrassem a relação do bruxismo em crianças com fatores psicológicos como ansiedade, estresse, distúrbios do humor e emoções.

Foram excluídos todos os estudos que incluíram na amostra crianças com síndromes congênitas e/ ou cromossômicas, alterações sistêmicas, paralisia cerebral e distúrbios neurológicos, bem como não foram admitidas as pesquisas que apresentavam metodologia delineada com resultados não condizentes com os objetivos, revisões, cartas ao editor, e, ainda, relato ou série de casos. Além disso, estudos com pacientes que apresentassem distúrbios do sono como síndrome da apneia obstrutiva crônica não foram considerados para esta revisão.

Inicialmente, os títulos e resumos dos artigos identificados por este processo foram avaliados por dois revisores independentes, para verificar se preenchiam os critérios para inclusão desta pesquisa. Caso houvesse discordância entre os resultados dos revisores, o artigo era lido na íntegra e um terceiro revisor 
Rios LT, Aguiar VNP, Machado FC, Rocha CT, Neves BG. Bruxismo infantil e sua associação com fatores psicológicos - revisão sistemática da literatura. Rev. Odontol. Univ. Cid. São Paulo 2018 jan-mar; 30(1): 64-76

ISSN 1983-5183

era solicitado para participar do processo. A decisão final sobre quais artigos seriam incluídos na pesquisa foi tomada por consenso. As referências citadas de todos os artigos selecionados foram verificadas para potenciais artigos adicionais, sendo realizado assim um cruzamento das referências bibliográficas dos trabalhos encontrados.

Para os estudos incluídos, os seguintes dados foram coletados: autor, ano de publicação, tipo de estudo, tamanho da amostra, idade dos participantes, diagnóstico de bruxismo, fatores avaliados, instrumentos de coleta de dados (por exemplo, testes psicológicos), resultados e conclusões relativas à associação entre o bruxismo em crianças e fatores psicológicos.

\section{RESULTADOS}

Um total de 97 publicações foi identificado na busca inicial. Após aplicação dos critérios de inclusão, a amostra final foi composta por sete estudos. A Figura 1 apresenta o fluxograma com a síntese do processo de seleção dos artigos.

Inicialmente, os artigos foram avaliados pelo título e resumo. Após a eliminação dos 31 artigos duplicados, foram selecionados 66 artigos. Desses, 59 foram excluídos, sendo 57 após a análise dos títulos e resumos, e dois foram excluídos após leitura do artigo na íntegra. A principal razão para exclusão foi a não relevância ao estudo ( $n=24)$, seguida por fugir dos critérios de inclusão $(n=18)$ (Figura 1). Após a seleção inicial, os estudos foram analisados por dois revisores que leram a versão na íntegra dos artigos. Mais uma vez, os artigos que não apresentavam o principal objetivo desta revisão e não se enquadravam aos critérios de inclusão foram excluídos $(n=2)$. As características e os resultados dos estudos incluídos nesta revisão estão detalhados no Quadro 1. 
Rios LT, Aguiar VNP, Machado FC, Rocha CT, Neves BG. Bruxismo infantil e sua associação com fatores psicológicos - revisão sistemática da literatura. Rev. Odontol. Univ. Cid. São Paulo 2018 jan-mar; 30(1): 64-76

ISSN 1983-5183

Figura 1. Fluxograma de identificação e seleção dos artigos para revisão sistemática sobre a associação de fatores psicológicos e bruxismo em crianças.

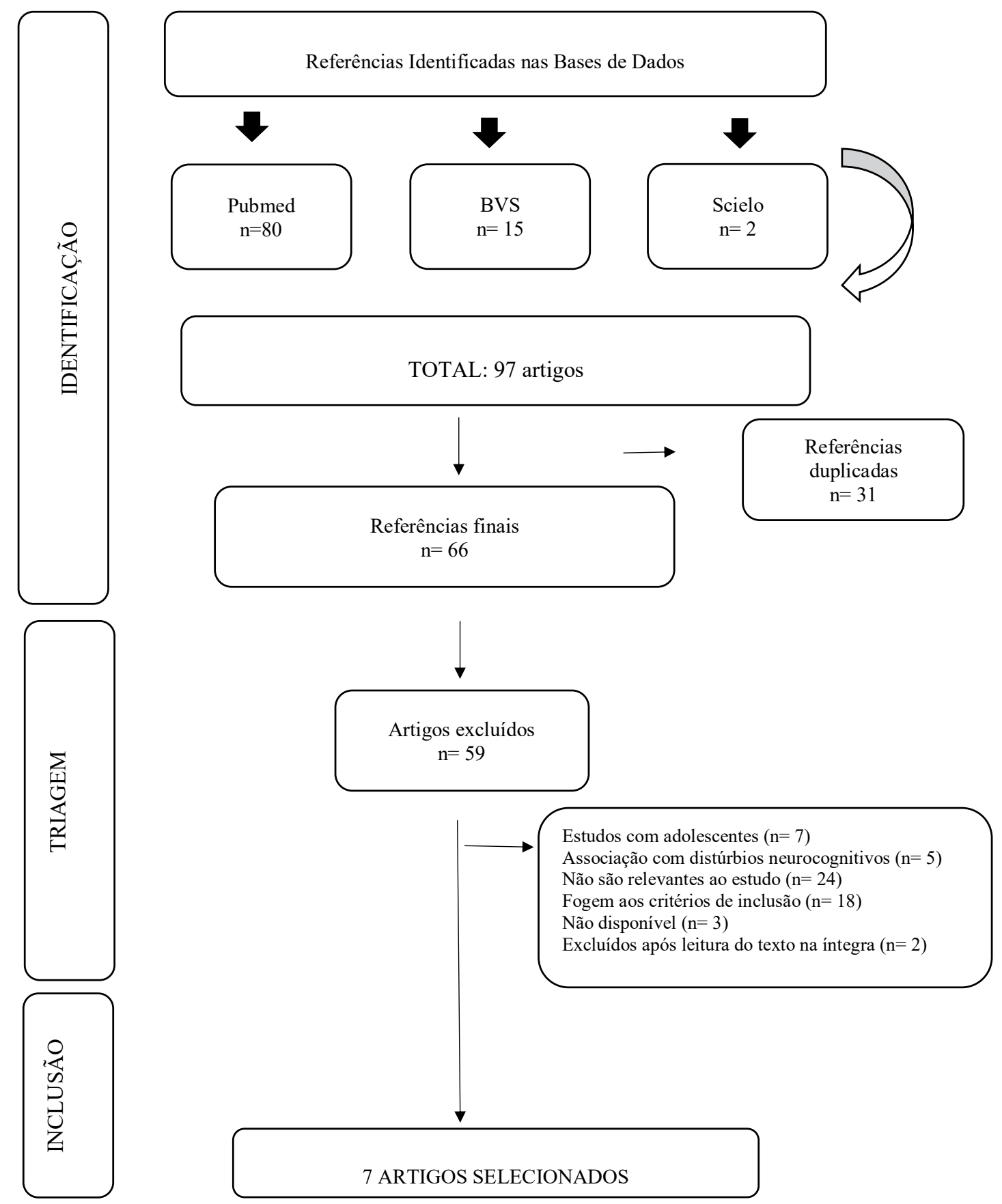

Quadro 1. Características dos estudos selecionados. 
Rios LT, Aguiar VNP, Machado FC, Rocha CT, Neves BG. Bruxismo infantil e sua associação com fatores psicológicos - revisão sistemática da literatura. Rev. Odontol. Univ. Cid. São Paulo 2018 jan-mar; 30(1): 64-76

\section{ISSN 1983-5183}

\begin{tabular}{|c|c|c|c|c|c|c|c|}
\hline $\begin{array}{l}\text { Autor/tipo de } \\
\text { estudo/local } \\
\text { do estudo }\end{array}$ & Amostra & Idade & $\begin{array}{l}\text { Diagnóstico de } \\
\text { bruxismo }\end{array}$ & $\begin{array}{l}\text { Fatores } \\
\text { avaliados }\end{array}$ & Instrumentos & Resultados & Conclusão \\
\hline $\begin{array}{l}\text { Retrespo et } \\
\text { al., } 2008^{15} \\
\text { Caso-controle } \\
\text { Medellin, } \\
\text { Colômbia }\end{array}$ & $\begin{array}{l}52 \text { crianças } \\
26 \text { bruxistas; } \\
\text { Segundo } \\
\text { critérios de } \\
\text { classificação } \\
\text { propostos } \\
\text { pela Academia } \\
\text { Americana de } \\
\text { Medicina do } \\
\text { Sono } \\
26 \text { grupo- } \\
\text {-controle }\end{array}$ & $\begin{array}{l}8 \text { e } 11 \\
\text { anos }\end{array}$ & $\begin{array}{l}\text { Baseado nos } \\
\text { critérios da } \\
\text { AAMS e exame } \\
\text { clínico para } \\
\text { avaliar desgaste } \\
\text { dental. }\end{array}$ & $\begin{array}{l}\text { O padrão de } \\
\text { persona- } \\
\text { lidade e } \\
\text { o nível de } \\
\text { ansiedade } \\
\text { de crianças } \\
\text { bruxistas em } \\
\text { compara- } \\
\text { ção com } \\
\text { o grupo- } \\
\text {-controle. } \\
\text { DTM } \\
\text { também } \\
\text { foi avaliada } \\
\text { usando os } \\
\text { Critérios de } \\
\text { diagnós- } \\
\text { tico para } \\
\text { desordens } \\
\text { temporo- } \\
\text { mandibu- } \\
\text { lares (RDC / } \\
\text { TMD). }\end{array}$ & $\begin{array}{l}\text { Questionário de } \\
\text { Personalidade de } \\
\text { Crianças (CPQ); } \\
\text { Conners'Escalas } \\
\text { de avaliação dos } \\
\text { pais (CPRS). }\end{array}$ & $\begin{array}{l}\text { O valor médio de an- } \\
\text { siedade, medido com } \\
\text { o CPRS foi maior para o } \\
\text { grupo Bruxista do que } \\
\text { para o grupo controle. } \\
\text { A personalidade com } \\
\text { traços de maior tensão } \\
\text { foi considerada como } \\
\text { um fator de risco para } \\
\text { adquirir bruxismo ao } \\
\text { passo que a personali- } \\
\text { dade com traços mais } \\
\text { relaxados foi consi- } \\
\text { derada um fator de } \\
\text { proteção ao bruxismo. } \\
\text { A presença de uma } \\
\text { condição de ansiedade } \\
\text { aumentou o risco de } \\
\text { ser um bruxista. }\end{array}$ & $\begin{array}{l}\text { O perfil da } \\
\text { personalidade } \\
\text { dos sujeitos } \\
\text { parecia ser } \\
\text { fortemente as- } \\
\text { sociado com o } \\
\text { comportamen- } \\
\text { to de bruxismo } \\
\text { nas crianças } \\
\text { estudadas. } \\
\text { Há uma pos- } \\
\text { sível relação } \\
\text { etiológica entre } \\
\text { a alta tensão, } \\
\text { traços de } \\
\text { personalidade, } \\
\text { bruxismo, e } \\
\text { DTM. }\end{array}$ \\
\hline $\begin{array}{l}\text { Serra-Negra et } \\
\text { al., } 2009^{18} \\
\text { Caso-controle } \\
\text { Belo Horizon- } \\
\text { te, Brasil }\end{array}$ & $\begin{array}{l}652 \text { crianças } \\
\text { selecionadas } \\
\text { aleatoriamen- } \\
\text { te de escolas } \\
\text { públicas e } \\
\text { privadas }\end{array}$ & $\begin{array}{l}7-10 \\
\text { anos }\end{array}$ & $\begin{array}{l}\text { O diagnóstico } \\
\text { do bruxismo foi } \\
\text { determinado } \\
\text { através de um } \\
\text { questionário } \\
\text { de acordo com } \\
\text { os critérios da } \\
\text { AASM. }\end{array}$ & $\begin{array}{l}\text { Prevalência } \\
\text { de bruxismo } \\
\text { do sono em } \\
\text { escolares } \\
\text { brasileiros, } \\
\text { associações } \\
\text { com fatores } \\
\text { sócio-de- } \\
\text { mográficos, } \\
\text { níveis de } \\
\text { estresse, e } \\
\text { traços de } \\
\text { personali- } \\
\text { dade. }\end{array}$ & $\begin{array}{l}\text { Questionário para } \\
\text { os pais, Escala de } \\
\text { Estresse Infantil, } \\
\text { e as escalas de } \\
\text { neuroticismo e } \\
\text { responsabilidade. } \\
\text { Testes psicológi- } \\
\text { cos foram aplica- } \\
\text { dos e avaliados } \\
\text { por psicólogos. O } \\
\text { Índice de Vulnera- } \\
\text { bilidade Social do } \\
\text { banco de dados } \\
\text { do conselho da } \\
\text { cidade foi usado } \\
\text { para determinar } \\
\text { classificação so- } \\
\text { cial das famílias. }\end{array}$ & $\begin{array}{l}\text { A prevalência de } \\
35,3 \% \text { de bruxismo foi } \\
\text { encontrada. Não foi } \\
\text { encontrada associa- } \\
\text { ção entre bruxismo e } \\
\text { estresse, sexo, idade ou } \\
\text { vulnerabilidade social. } \\
\text { Crianças com altos } \\
\text { níveis de neuroticismo } \\
\text { (OR = 1,9, IC 1.3- } 2.6) \text { e } \\
\text { responsabilidade (OR = } \\
2,2, \text { IC } 1,0-5,0) \text { são duas } \\
\text { vezes mais propensos } \\
\text { a ter o hábito de bru- } \\
\text { xismo do sono quando } \\
\text { comparados com } \\
\text { aqueles que têm bai- } \\
\text { xos níveis desses traços } \\
\text { de personalidade. }\end{array}$ & $\begin{array}{l}\text { Um alto grau } \\
\text { de respon- } \\
\text { sabilidade e } \\
\text { neuroticismo, } \\
\text { que são traços } \\
\text { de personalida- } \\
\text { de individuais, } \\
\text { são fatores } \\
\text { determinantes } \\
\text { para o desen- } \\
\text { volvimento do } \\
\text { bruxismo do } \\
\text { sono entre as } \\
\text { crianças. }\end{array}$ \\
\hline $\begin{array}{l}\text { Ferreira-Bacci } \\
\text { et al., } 2012^{13} \\
\text { Ribeirão Preto, } \\
\text { Brasil }\end{array}$ & $\begin{array}{l}29 \text { crianças ( } 18 \\
\text { do sexo mas- } \\
\text { culino e } 11 \\
\text { do feminino) } \\
\text { cujos pais ou } \\
\text { responsáveis } \\
\text { relataram que } \\
\text { as mesmas } \\
\text { apresentavam } \\
\text { rangimento e/ } \\
\text { ou aperta- } \\
\text { mento dental } \\
\text { frequente. } \\
\text { Sem grupo- } \\
\text {-controle. }\end{array}$ & $\begin{array}{l}\text { 7- } 11 \\
\text { anos }\end{array}$ & $\begin{array}{l}\text { Estabelecido } \\
\text { com base no } \\
\text { relatório dos } \\
\text { pais/ respon- } \\
\text { sáveis sobre o } \\
\text { comportamen- } \\
\text { to das crianças } \\
\text { e possíveis } \\
\text { desordens do } \\
\text { sistema esto- } \\
\text { matognático } \\
\text { aliadas à pre- } \\
\text { sença de sinais } \\
\text { e sintomas de } \\
\text { bruxismo. }\end{array}$ & $\begin{array}{l}\text { Perfil com- } \\
\text { portamental } \\
\text { de um } \\
\text { grupo de } \\
\text { crianças com } \\
\text { bruxismo. } \\
\text { Estresse/ } \\
\text { ansiedade, } \\
\text { distúrbios } \\
\text { psicológicos } \\
\text { e de perso- } \\
\text { nalidade }\end{array}$ & $\begin{array}{l}\text { Relatos dos pais/ } \\
\text { responsáveis, } \\
\text { avaliação clínica, } \\
\text { Escala Comporta- } \\
\text { mental Infantil A2 } \\
\text { de Rutter, Escala } \\
\text { de Stress Infantil. }\end{array}$ & $\begin{array}{l}\text { Vinte e quatro (82,76\%) } \\
\text { crianças necessitavam } \\
\text { de intervenção psico- } \\
\text { lógica ou psiquiátrica, } \\
17 \text { destas crianças } \\
\text { apresentavam desor- } \\
\text { dens neurológicas } \\
\text { e } 7 \text { apresentavam } \\
\text { desordens } \\
\text { anti-sociais, } 6 \text { (20,70\%) } \\
\text { do total de crianças } \\
\text { apresentaram manifes- } \\
\text { tações } \\
\text { físicas e psicológicas } \\
\text { de estresse. }\end{array}$ & $\begin{array}{l}\text { Problemas } \\
\text { comportamen- } \\
\text { tais e emocio- } \\
\text { nais podem ser } \\
\text { fatores de ris- } \\
\text { cos potenciais } \\
\text { para bruxismo } \\
\text { em crianças. }\end{array}$ \\
\hline
\end{tabular}


Rios LT, Aguiar VNP, Machado FC, Rocha CT, Neves BG. Bruxismo infantil e sua associação com fatores psicológicos - revisão sistemática da literatura. Rev. Odontol. Univ. Cid. São Paulo 2018 jan-mar; 30(1): 64-76

ISSN 1983-5183

\begin{tabular}{|c|c|c|c|c|c|c|c|}
\hline $\begin{array}{l}\text { Autor/tipo de } \\
\text { estudo/local } \\
\text { do estudo }\end{array}$ & Amostra & Idade & $\begin{array}{l}\text { Diagnóstico de } \\
\text { bruxismo }\end{array}$ & $\begin{array}{l}\text { Fatores } \\
\text { avaliados }\end{array}$ & Instrumentos & Resultados & Conclusão \\
\hline $\begin{array}{l}\text { Renner et al, } \\
2012^{14} \\
\text { Coorte } \\
\text { São Paulo, } \\
\text { Brasil } \\
\text { Maranhão, } \\
\text { Brasil }\end{array}$ & $\begin{array}{l}869 \text { crianças } \\
\text { em Ribeirão } \\
\text { Preto (RP) e } \\
805 \text { crianças } \\
\text { em São Luís } \\
\text { (SL), Brasil }\end{array}$ & $\begin{array}{l}9-11 \\
\text { anos (RP) } \\
7-9 \text { anos } \\
\text { (SL) }\end{array}$ & $\begin{array}{l}\text { Questionário } \\
\text { aplicado aos } \\
\text { pais / respon- } \\
\text { sáveis para } \\
\text { determinar } \\
\text { diagnóstico do } \\
\text { bruxismo. }\end{array}$ & $\begin{array}{l}\text { Prevalência } \\
\text { de bruxismo } \\
\text { e suas asso- } \\
\text { ciações com } \\
\text { problemas } \\
\text { mentais e } \\
\text { depressão. }\end{array}$ & $\begin{array}{l}\text { Questionário de } \\
\text { saúde e socio- } \\
\text {-demográfico, } \\
\text { Strength and } \\
\text { Difficulties Ques- } \\
\text { tionnaire para } \\
\text { avaliação dos } \\
\text { problemas men- } \\
\text { tais e o Children's } \\
\text { Depression } \\
\text { Inventory para } \\
\text { depressão. }\end{array}$ & $\begin{array}{l}\text { Na análise univariada, } \\
\text { problemas de saúde } \\
\text { mental, de conduta, } \\
\text { sintomas emocionais } \\
\text { e hiperatividade foram } \\
\text { associados com uma } \\
\text { alta prevalência de } \\
\text { bruxismo. A análise } \\
\text { multivariada identifi- } \\
\text { cou uma significativa } \\
\text { associação do bru- } \\
\text { xismo com sintomas } \\
\text { emocionais. }\end{array}$ & $\begin{array}{l}\text { Sintomas } \\
\text { emocionais } \\
\text { e problemas } \\
\text { de saúde } \\
\text { mental foram } \\
\text { associados à } \\
\text { alta prevalência } \\
\text { de bruxismo. } \\
\text { Não houve } \\
\text { associação da } \\
\text { depressão com } \\
\text { o bruxismo. }\end{array}$ \\
\hline $\begin{array}{l}\text { Serra-Negra et } \\
\text { al., } 2012^{17} \\
\text { Caso-controle } \\
\text { Belo Horizon- } \\
\text { te, Brasil. }\end{array}$ & $\begin{array}{l}\text { Um total de } \\
360 \text { crianças } \\
\text { em idade } \\
\text { escolar (casos } \\
120 \text { e } 240 \\
\text { controles) } \\
\text { pareados por } \\
\text { idade, sexo e } \\
\text { nível socioeco- } \\
\text { nômico }\end{array}$ & $\begin{array}{l}\text { 7- } 11 \\
\text { anos }\end{array}$ & $\begin{array}{l}\text { Questionário } \\
\text { baseado no } \\
\text { relato dos pais } \\
\text { e de acordo } \\
\text { com os critérios } \\
\text { da AASM. }\end{array}$ & $\begin{array}{l}\text { Associação } \\
\text { entre os } \\
\text { níveis de es- } \\
\text { tresse, traços } \\
\text { de perso- } \\
\text { nalidade e } \\
\text { bruxismo } \\
\text { em crianças.. }\end{array}$ & $\begin{array}{l}\text { Questionário } \\
\text { aplicado aos pais; } \\
\text { escala de estresse } \\
\text { infantil e escala } \\
\text { de neuroticismo e } \\
\text { responsabilidade } \\
\text { aplicado para as } \\
\text { crianças (versão } \\
\text { brasileira). Testes } \\
\text { psicológicos } \\
\text { foram aplicados } \\
\text { e avaliados por } \\
\text { psicólogos }\end{array}$ & $\begin{array}{l}\text { Crianças com alto } \\
\text { nível de estresse (odds } \\
\text { ratio=1.8; intervalo de } \\
\text { confiança=1.1-2.9) e } \\
\text { elevado senso de res- } \\
\text { ponsabilidade (OR=1.6; } \\
\mathrm{Cl}=1.0-2.5 \text { ) apresentam } \\
\text { quase duas vezes mais } \\
\text { chances de apresentar } \\
\text { o hábito de bruxismo. }\end{array}$ & $\begin{array}{l}\text { Sugere haver } \\
\text { uma relação } \\
\text { direta entre a } \\
\text { presença do } \\
\text { transtorno de } \\
\text { ansiedade e o } \\
\text { estabelecimen- } \\
\text { to do bruxismo } \\
\text { em crianças. }\end{array}$ \\
\hline $\begin{array}{l}\text { Serra-Negra, } \\
\text { et al, } 2013^{19} \\
\text { Caso-controle } \\
\text { Belo Horizon- } \\
\text { te, Brasil. }\end{array}$ & $\begin{array}{l}652 \text { crianças } \\
\text { selecionadas } \\
\text { aleatoriamen- } \\
\text { te de escolas } \\
\text { públicas e pri- } \\
\text { vadas de Belo } \\
\text { Horizonte, } \\
\text { Brasil }\end{array}$ & 7-10 anos & $\begin{array}{l}\text { Questionário } \\
\text { de acordo com } \\
\text { os critérios da } \\
\text { AASM. }\end{array}$ & $\begin{array}{l}\text { Associação } \\
\text { entre as } \\
\text { tarefas das } \\
\text { crianças, tra- } \\
\text { ços de per- } \\
\text { sonalidade e } \\
\text { bruxismo do } \\
\text { sono }\end{array}$ & $\begin{array}{l}\text { Questionário para } \\
\text { os pais e crian- } \\
\text { ças, Escala de } \\
\text { Estresse Infantil, } \\
\text { e as escalas de } \\
\text { neuroticismo e } \\
\text { responsabilidade. } \\
\text { Testes psicológi- } \\
\text { cos foram aplica- } \\
\text { dos e avaliados } \\
\text { por psicólogos. O } \\
\text { Índice de Vulnera- } \\
\text { bilidade Social do } \\
\text { banco de dados } \\
\text { do conselho da } \\
\text { cidade foi usado } \\
\text { para determinar } \\
\text { classificação so- } \\
\text { cial das famílias. }\end{array}$ & $\begin{array}{l}\text { A maioria das famílias } \\
\text { foram classificadas } \\
\text { como tendo baixa } \\
\text { vulnerabilidade social } \\
(61,3 \%) .(56,4 \%) \text { das } \\
\text { meninas realizavam } \\
\text { trabalho doméstico e } \\
\text { alguma atividade artís- } \\
\text { tica (51,3\%), enquanto } \\
\text { as atividades desporti- } \\
\text { vas eram mais comuns } \\
\text { entre os rapazes (61\%). } \\
\text { O bruxismo do sono } \\
\text { foi mais prevalente em } \\
\text { crianças que marcaram } \\
\text { altamente na sub-es- } \\
\text { cala de neuroticismo e } \\
\text { que, frequentemente, } \\
\text { realizavam tarefas } \\
\text { domésticas. }\end{array}$ & $\begin{array}{l}\text { As crianças } \\
\text { cujas perso- } \\
\text { nalidades têm } \\
\text { um alto nível } \\
\text { de neuroti- } \\
\text { cismo e que } \\
\text { realizam tarefas } \\
\text { domésticas } \\
\text { impostas pela } \\
\text { família são mais } \\
\text { vulneráveis ao } \\
\text { bruxismo do } \\
\text { sono. }\end{array}$ \\
\hline
\end{tabular}


Rios LT, Aguiar VNP, Machado FC, Rocha CT, Neves BG. Bruxismo infantil e sua associação com fatores psicológicos - revisão sistemática da literatura. Rev. Odontol. Univ. Cid. São Paulo 2018 jan-mar; 30(1): 64-76

\section{ISSN 1983-5183}

\begin{tabular}{|c|c|c|c|c|c|c|c|}
\hline $\begin{array}{l}\text { Autor/tipo de } \\
\text { estudo//ocal } \\
\text { do estudo }\end{array}$ & Amostra & Idade & $\begin{array}{l}\text { Diagnóstico de } \\
\text { bruxismo }\end{array}$ & $\begin{array}{l}\text { Fatores } \\
\text { avaliados }\end{array}$ & Instrumentos & Resultados & Conclusão \\
\hline $\begin{array}{l}\text { Oliveira et al., } \\
2015^{16} \\
\text { Caso- controle } \\
\text { Santa Catari- } \\
\text { na, Brasil. }\end{array}$ & $\begin{array}{l}\text { Grupo com } \\
\text { bruxismo (BG): } \\
42 \text { crianças } \\
\text { de ambos os } \\
\text { sexos } \\
\text { Grupo-con- } \\
\text { trole - sem } \\
\text { bruxismo : } 42 \\
\text { crianças de } \\
\text { ambos sexos. }\end{array}$ & $6-8$ anos & $\begin{array}{l}\text { Critérios míni- } \\
\text { mos propostos } \\
\text { pela Academia } \\
\text { Americana de } \\
\text { Medicina do } \\
\text { Sono (AAMS) e } \\
\text { exame clínico } \\
\text { para verificar } \\
\text { o desgaste } \\
\text { dentário. }\end{array}$ & $\begin{array}{l}\text { Nivel de } \\
\text { ansiedade } \\
\text { em crianças } \\
\text { com e sem } \\
\text { bruxismo }\end{array}$ & $\begin{array}{l}\text { Entrevista com } \\
\text { pais/ responsá- } \\
\text { veis sobre hábitos } \\
\text { e comportamen- } \\
\text { tos de seus filhos, } \\
\text { escala de estado/ } \\
\text { traço de ansieda- } \\
\text { de proposta por } \\
\text { Bouden. }\end{array}$ & $\begin{array}{l}\text { O grupo BG, de acordo } \\
\text { com a observação de } \\
\text { seus pais/responsá- } \\
\text { veis, apresentou uma } \\
\text { proporção maior de } \\
\text { crianças ansiosas } \\
(p=0,001 \text { ) e nervosas } \\
(p=0,043) \text {. Nenhuma } \\
\text { diferença significativa } \\
\text { foi observada em } \\
\text { relação à timidez ( } p= \\
0,056) \text { e agressivida- } \\
\text { de ( } p=0,155 \text { ) entre } \\
\text { os grupos. Escala de } \\
\text { ansiedade aplicada } \\
\text { revelou diferença entre } \\
\text { os grupos ( } p=0,0136 \text { ) } \\
\text { com maiores níveis } \\
\text { de ansiedade para o } \\
\text { grupo BG. }\end{array}$ & $\begin{array}{l}\text { Crianças } \\
\text { bruxômeras são } \\
\text { mais inquietas, } \\
\text { preocupadas } \\
\text { e ansiosas, } \\
\text { indicando } \\
\text { uma relação } \\
\text { direta entre a } \\
\text { presença de } \\
\text { transtorno de } \\
\text { ansiedade e o } \\
\text { estabelecimen- } \\
\text { to de bruxismo } \\
\text { em crianças. }\end{array}$ \\
\hline
\end{tabular}

O tamanho das amostras avaliadas nos estudos variou de 29 a 869 crianças, com idade entre seis a onze anos. Os estudos selecionados foram realizados em dois países: Brasil e Colômbia, sendo todos publicados na língua inglesa.

Ferreira-Bacci et al. ${ }^{13}$ (2012) constataram que aproximadamente $82,76 \%$ das crianças com bruxismo apresentavam problemas emocionais ou de comportamento, necessitando de algum tipo de intervenção psicológica e/ou psiquiátrica. A análise dos escores da Escala de Stress Infantil aplicada às crianças mostrou que seis crianças $(20,70 \%)$ apresentaram manifestações físicas e psicológicas significativas de estresse caracterizadas por ranger de dentes, dores nas pernas, dor de estômago, enurese, se sentindo nervoso, preocupado, mal-humorado ou infeliz, distúrbios de medo, de sono, entre outras manifestações.

Já Renner et al..$^{14}$ (2012) realizaram seu estudo em duas cidades brasileiras: Ribeirão Preto - SP e São Luís - MA, encontrando uma alta prevalência de bruxismo em pacientes infantis acometidos por medos, tensões e ansiedade em ambas as cidades. Os autores constataram, ainda, associação entre o trabalho materno fora de casa e a cor da pele branca com a maior propensão do aparecimento de bruxismo.

Restrepo et al..$^{15}$ (2008) identificaram uma diferença significativa acerca de tensão e ansiedade entre os grupos-controle e o de crianças bruxômeras, sendo que estas apresentavam maiores níveis de tais traços de personalidade e de ansiedade do que aquelas que não apresentavam a parafunção.

No estudo de Oliveira et al. ${ }^{16}$ (2015), não foi encontrada diferença significativa entre agressividade e timidez entre crianças bruxômeras e o grupo-controle. Nesse estudo, foram incluídas somente crianças na fase de dentição mista. Ao se analisar cada item da escala aplicada para avaliação de ansiedade, verificouse que as crianças com bruxismo eram mais inquietas, tinham maiores preocupações sobre a escola e apresentavam uma maior quantidade de queixas espontâneas de esquecimento e lacunas de memória do que os indivíduos do grupo-controle, o que sugere uma associação entre esses fatores e bruxismo.

Em um modelo para avaliação das características de personalidade, neuroticismo e responsabilidade foram considerados importantes nos estudos de Serra-Negra et al. ${ }^{17}$, (2012) e de Serra-Negra et al. ${ }^{18}$ (2009), sendo a primeira relacionada à vulnerabilidade a emoções negativas, ansiedade, raiva, culpa e depressão 
Rios LT, Aguiar VNP, Machado FC, Rocha CT, Neves BG. Bruxismo infantil e sua associação com fatores psicológicos - revisão sistemática da literatura. Rev. Odontol. Univ. Cid. São Paulo 2018 jan-mar; 30(1): 64-76

ISSN 1983-5183

clínica, já a responsabilidade foi relacionada com a autodisciplina, comportamento obediente e esforço para sucesso. Altos níveis de estresse e de responsabilidade foram identificados como fatores potenciais que podem contribuir para a ocorrência de bruxismo em crianças, sendo considerado um mecanismo liberador de tensões. ${ }^{17}$

Serra-Negra et al. ${ }^{18}$ (2009) observaram que crianças bruxômeras apresentavam alto grau de neuroticismo e que crianças com alto senso de responsabilidade tendem a desenvolver bruxismo ${ }^{17}$. Já em um outro estudo de um mesmo grupo de pesquisa ${ }^{19}$, foi confirmado o dado encontrado em seu estudo de 2009 , que crianças que realizavam tarefas domésticas eram mais susceptíveis a desenvolver bruxismo ${ }^{18}$.

\section{DISCUSSÃO}

Nesta revisão sistemática, foram investigadas as potenciais associações entre o bruxismo e fatores psicológicos em crianças. Devido ao crescente aumento de casos de bruxismo infantil, vários autores investigaram uma relação entre esta desordem e fatores psicológicos, tais como ansiedade e estresse ${ }^{13,}$ $16,18,20,21,22,23$. Estudos já mostraram que crianças com problemas psicológicos apresentam de 36 a $40 \%$ de chance de desenvolver bruxismo ${ }^{24}$.

A avaliação de ansiedade e estresse infantil é um desafio, uma vez que a maioria das crianças não sabe o significado de ansiedade ou mesmo relatar como se sente ao estar ansiosa ${ }^{25}$. A ansiedade consiste em uma sensação desagradável, caracterizada por preocupação, tensão e medo e pode ser sentida em níveis variáveis ${ }^{9,26}$. É mais provável que seja subdiagnosticada por apresentar sintomatologia diversa, particularmente de acordo com as diferentes fases de desenvolvimento. Além disso, as ferramentas disponíveis para avaliar a ansiedade parecem implicar problemas de confiabilidade evalidade ${ }^{16}$. Questionários de avaliação de personalidade e ansiedade têm sido fortemente criticados, por muitas razões: capacidade de falsa resposta, influência de estilo de resposta, ausência do entendimento das próprias sensações e variação entre diferentes instrumentos de análise ${ }^{27}$.

A capacidade para lidar com estresse é única para cada indivíduo e pode estar diretamente relacionada com a formação da personalidade. Crianças bruxômeras, além da ansiedade já relatada, também apresentam traços de personalidade específicos, como agressividade, neuroticismo, problemas comportamentais e desequilíbrios emocionais..$^{15}$ No estudo de Insana et al..$^{28}$ (2013), observou-se que o bruxismo foi associado com comportamentos de internalização ${ }^{28}$. Renner et al. ${ }^{14}$ (2012) não identificaram associação da depressão com o bruxismo ${ }^{14}$. Em um trabalho incluindo adultos, foram encontrados resultados semelhantes quando comparados com aqueles incluindo crianças para avaliação de transtornos de ansiedade, transtorno bipolar e depressão, os quais são mais frequentes em indivíduos com bruxismo quando comparados aos indivíduos sem bruxismo ${ }^{29}$. No entanto, ainda existem poucos dados disponíveis acerca da relação entre bruxismo do sono e sintomas psiquiátricos em crianças e adolescentes ${ }^{23}$.

No geral, apesar de diferentes critérios de avaliação entre os estudos desta revisão, vários identificaram uma associação do bruxismo com ansiedade ${ }^{20,21,25,26}$ e com o estresse $e^{13,17,23}$. Nos estudos de Serra-Negra et al. ${ }^{17}$, (2012) e de Serra-Negra et al..$^{18}$ (2009), a avaliação da ansiedade e estresse foi baseada na Child Stress Scale aplicada com crianças, já o estudo de Oliveira et al. ${ }^{16}$ (2015), utilizou como ferramenta para esta avaliação a escala de traço de ansiedade para crianças proposta por Bouden et al. ${ }^{30}$ (2000), e aplicada com os pais ou responsáveis. Embora existam escalas para quantificar o nível de ansiedade em crianças, elas não deveriam ser utilizadas de maneira isolada, sendo importante o relato dos pais e de cuidadores ${ }^{16}$. Dessa forma, algumas 
Rios LT, Aguiar VNP, Machado FC, Rocha CT, Neves BG. Bruxismo infantil e sua associação com fatores psicológicos - revisão sistemática da literatura. Rev. Odontol. Univ. Cid. São Paulo 2018 jan-mar; 30(1): 64-76

ISSN 1983-5183

limitações metodológicas desta revisão devem ser consideradas, uma vez que não houve padronização nos diferentes estudos selecionados desta revisão quanto ao instrumento de pesquisa utilizado para quantificar os fatores psicológicos, dificultando, assim, o estabelecimento de parâmetros comparativos.

Acredita-se que os indivíduos com bruxismo desenvolvem este hábito por causa da influência de fatores emocionais, como ter que lidar com um acúmulo de tarefas, perdas, expectativas, conflitos de autoimagem, autoestima e ansiedade ${ }^{15,17,19}$. De acordo com a avaliação de cada item da escala State-Trait Anxiety Inventory for Children (STAIC), observou-se que crianças com bruxismo são mais nervosas, ansiosas e apresentam maiores queixas de esquecimento espontâneo ou lacunas de memória ${ }^{16}$, o que corrobora estudos anteriores que sugerem que crianças que sofrem cobrança em demasia, com pais mais rigorosos, tendem a desenvolver mecanismos de defesa emocional, facilitando o aparecimento de hábitos destrutivos, como o apertar e/ou ranger os dentes ${ }^{18,31}$.

As atividades exercidas e o ambiente onde a criança está inserida também têm influência sobre a condição de ansiedade e estresse ${ }^{14,18}$. De acordo com Renner et al. ${ }^{14}$ (2012), o trabalho materno fora de casa foi um fator associado com o aumento da prevalência de bruxismo em seu estudo. Outros fatores como renda familiar e pais divorciados também têm sido um fator preditivo para o aparecimento de bruxismo infanti $^{19}$.

Outra limitação dos estudos que avaliam a relação entre bruxismo e fatores psicológicos é a subjetividade do diagnóstico de bruxismo. A maioria dos dados sobre a associação entre transtornos psicossociais e bruxismo vem de estudos que adotam um diagnóstico clínico e/ou auto-relato de bruxismo. Em geral, esses tipos de estudo mostraram algum tipo de associação de bruxismo com ansiedade, sensibilidade ao estresse, depressão e outras características de personalidade, diferindo das investigações laboratoriais do sono ${ }^{20}$. Cabe ressaltar que alguns estudos selecionados desta revisão avaliaram a presença ou ausência do bruxismo baseado no relato dos pais de acordo com os critérios da Associação Americana de Medicina do Sono ${ }^{13-19}$. No entanto, outros estudos também avaliaram critérios clínicos para identificar desgaste dentário ${ }^{15,16 .}$

A manutenção desse hábito na vida adulta pode comprometer a saúde bucal ${ }^{18}$. No entanto, ainda são inconclusivos os estudos que relatam os prejuízos advindos da manutenção do bruxismo infantil na vida adulta, uma vez que pesquisas acerca desse hábito na infância vêm ganhando maior importância nos últimos anos ${ }^{32}$. Em crianças mais novas, o bruxismo pode ser uma consequência da imaturidade do sistema neuromuscular mastigatório. A instabilidade da oclusão durante a troca da dentição decídua pela dentição permanente é outro fator etiológico que pode estar relacionado com o bruxismo em crianças ${ }^{9}$. Geralmente, é mais severo em crianças em idade pré-escolar, devido às características estruturais e morfofuncionais dos dentes decíduos, embora sua maior ocorrência seja na fase de dentição mista, devido às interferências oclusais se desenvolverem naturalmente durante a erupção dos dentes ${ }^{33}$. A idade das crianças avaliadas nos estudos desta revisão variou de seis a onze anos, idade que coincide com a fase de dentição mista.

Torna-se de grande valia o conhecimento das possíveis etiologias dessa parafunção e, quando detectada a origem psicológica, essas crianças devem ser acompanhadas para que intervenções apropriadas sejam realizadas ${ }^{34}$. Ressalta-se que uma intervenção psicológica pode promover uma melhor chance de lidar com estímulos externos para as crianças com um elevado nível de responsabilidade e uma alta tendência para o estresse, que pode, por sua vez, oferecer-lhes uma melhor chance de se tornar adultos mais emocionalmente saudáveis ${ }^{17}$. A associação entre traços de personalidade e hábito de bruxismo na 
Rios LT, Aguiar VNP, Machado FC, Rocha CT, Neves BG. Bruxismo infantil e sua associação com fatores psicológicos - revisão sistemática da literatura. Rev. Odontol. Univ. Cid. São Paulo 2018 jan-mar; 30(1): 64-76

ISSN 1983-5183

infância sugere que o tratamento psicológico durante a infância pode permitir que os indivíduos possam encarar o conflito ou tensão e ter um efeito sobre o controle do hábito ${ }^{18 .}$

Tendo-se em vista que odontopediatras estão entre os primeiros profissionais a serem procurados pelos pais e crianças quanto à queixa do bruxismo, eles devem ser aptos a identificar suas possíveis causas, características clínicas e seus sinais e sintomas, devendo o problema ser identificado o mais precoce possível. Quando diagnosticada a origem psicológica do bruxismo, o paciente deve ser encaminhado a um profissional especializado, sendo de fundamental importância a interação multiprofissional no tratamento dessa parafunção, sendo este um dos paradigmas para um tratamento eficaz.

\section{CONCLUSÃO}

Com base nas evidências encontradas, há uma associação significativa entre estresse, ansiedade e fatores psicológicos com o bruxismo infantil. No entanto, ainda existe uma enorme necessidade de estudos bem delineados e com metodologias padronizadas a fim de verificar a associação entre o bruxismo e fatores psicológicos em crianças.

\section{REFERÊNCIAS}

1. AMERICAN Academy of Sleep. Medicine. The international classification of sleep disorders : diagnostic \& coding manual. Westchester, IL: American Academy of Sleep Medicine; 2005.

2. PIZZOL KEDC, Carvalho JCQ, Konishi F, Marcomini EMS, Giusti JSM. Bruxismo na infância: fatores etiológicos e possíveis tratamentos. Rev odontol UNESP 2006 35(2):157-63.

3. CARRA MC, Bruni O, Huynh N. Topical review: sleep bruxism, headaches, and sleep-disordered breathing in children and adolescents. Journal of orofacial pain 2012 Fall;26(4):267-76.

4. CARRA MC, Huynh N, Morton P, Rompre PH, Papadakis A, Remise C, et al. Prevalence and risk factors of sleep bruxism and wake-time tooth clenching in a 7- to 17-yr-old population. European journal of oral sciences 2011 Oct;119(5):386-94.

5. CHEIFETZ AT, Osganian SK, Allred EN, Needleman HL. Prevalence of bruxism and associated correlates in children as reported by parents. Journal of dentistry for children (Chicago, III) 2005 May-Aug;72(2):67-73.

6. MANFREDINI D, Landi N, Romagnoli M, Bosco M. Psychic and occlusal factors in bruxers. Australian dental journal 2004 Jun;49(2):84-9.

7. VALERA FC, Travitzki LV, Mattar SE, Matsumoto MA, Elias AM, Anselmo-Lima WT. Muscular, functional and orthodontic changes in pre school children with enlarged adenoids and tonsils. International journal of pediatric otorhinolaryngology $2003 \mathrm{Jul} ; 67(7): 761-70$.

8. MACHADO E, Dal-Fabbro C, Cunali PA, Kaizer OB. Prevalence of sleep bruxism in children: a systematic review. Dental Press J Orthod 2014 dez;19(6):54-61.

9. BARBOSA Tde S, Miyakoda LS, Pocztaruk Rde L, Rocha CP, Gaviao MB. Temporomandibular disorders and bruxism in childhood and adolescence: review of the literature. International journal of pediatric otorhinolaryngology 2008 Mar;72(3):299-314. 
Rios LT, Aguiar VNP, Machado FC, Rocha CT, Neves BG. Bruxismo infantil e sua associação com fatores psicológicos - revisão sistemática da literatura. Rev. Odontol. Univ. Cid. São Paulo 2018 jan-mar; 30(1): 64-76

ISSN 1983-5183

10. LOBBEZOO F, Van Der Zaag J, Naeije M. Bruxism: its multiple causes and its effects on dental implants - an updated review. Journal of oral rehabilitation 2006 Apr;33(4):293300.

11. ALENCAR NA, Leao CS, Leao ATT, Luiz RR, Fonseca-Goncalves A, Maia LC. Sleep bruxism and anxiety impacts in quality of life related to oral health of Brazilian children and their families. The Journal of clinical pediatric dentistry 201741 (3):179-85.

12. TAKEMURA T, Takahashi T, Fukuda M, Ohnuki T, Asunuma T, Masuda Y, et al. A psychological study on patients with masticatory muscle disorder and sleep bruxism. Cranio : the journal of craniomandibular practice $2006 \mathrm{Jul} ; 24(3): 191-6$.

13. FERREIRA-BACCI Ado V, Cardoso CL, Diaz-Serrano KV. Behavioral problems and emotional stress in children with bruxism. Brazilian dental journal 2012 23(3):246-51.

14. RENNER AC, Da Silva AA, Rodriguez JD, Simoes VM, Barbieri MA, Bettiol H, et al. Are mental health problems and depression associated with bruxism in children? Community dentistry and oral epidemiology 2012 Jun;40(3):277-87.

15. RESTREPO CC, Vasquez LM, Alvarez M, Valencia I. Personality traits and temporomandibular disorders in a group of children with bruxing behaviour. Journal of oral rehabilitation 2008 Aug;35(8):585-93.

16. OLIVEIRA MT, Bittencourt ST, Marcon K, Destro S, Pereira JR. Sleep bruxism and anxiety level in children. Brazilian oral research 201529

17. SERRA-NEGRA JM, Paiva SM, Flores-Mendoza CE, Ramos-Jorge ML, Pordeus IA. Association among stress, personality traits, and sleep bruxism in children. Pediatric dentistry 2012 Mar-Apr;34(2):e30-4.

18. SERRA-NEGRA JM, Ramos-Jorge ML, Flores-Mendoza CE, Paiva SM, Pordeus IA. Influence of psychosocial factors on the development of sleep bruxism among children. International journal of paediatric dentistry 2009 Sep;19(5):309-17.

19. SERRA-NEGRA JM, Paiva SM, Abreu MH, Flores-Mendoza CE, Pordeus IA. Relationship between tasks performed, personality traits, and sleep bruxism in Brazilian school children--a population-based cross-sectional study. PloS one 2013 8(11):e80075.

20. MANFREDINI D, Lobbezoo F. Role of psychosocial factors in the etiology of bruxism. Journal of orofacial pain 2009 Spring;23(2):153-66.

21. GUNGORMUS Z, Erciyas K. Evaluation of the relationship between anxiety and depression and bruxism. The Journal of international medical research 2009 Mar-Apr;37(2):547-50.

22. KANEHIRA H, Agariguchi A, Kato H, Yoshimine $S$, Inoue $H$. Association between stress and temporomandibular disorder. Nihon Hotetsu Shika Gakkai zasshi 2008 Jul;52(3):37580.

23. TURKOGLU S, Akca OF, Turkoglu G, Akca M. Psychiatric disorders and symptoms in children and adolescents with sleep bruxism. Sleep \& breathing = Schlaf \& Atmung 2014 Sep;18(3):649-54. 
ISSN 1983-5183

24. HERRERA M, Valencia I, Grant M, Metroka D, Chialastri A, Kothare SV. Bruxism in children: effect on sleep architecture and daytime cognitive performance and behavior. Sleep 2006 Sep;29(9):1143-8.

25. MONACO A, Ciammella NM, Marci MC, Pirro R, Giannoni M. The anxiety in bruxer child. A case-control study. Minerva stomatologica 2002 Jun;51(6):247-50.

26. ASSUMPÇÃO Jr. FB, Resch CR. Trait anxiety evaluation scale validation: a study of sensibility and specifity. Braz J Psych 2006 10(1):19-25.

27. KAMPE T, Edman G, Bader G, Tagdae T, Karlsson S. Personality traits in a group of subjects with long-standing bruxing behaviour. Journal of oral rehabilitation 1997 Aug;24(8):588-93.

28. INSANA SP, Montgomery-Downs HE. Sleep and sleepiness among first-time postpartum parents: a field- and laboratory-based multimethod assessment. Developmental psychobiology 2013 May;55(4):361-72.

29. OHAYON MM, Li KK, Guilleminault C. Risk factors for sleep bruxism in the general population. Chest 2001 Jan;119(1):53-61.

30. BOUDEN A, Halayem MB, Fakhfakh R. Étude préliminaire de validation d'une échelle d'anxieté-trait chez l'enfant. Neuropsychiatr Enfance Adolesc 2000 50(2):25.

31. ANTONIO AG, Pierro VS, Maia LC. Bruxism in children: a warning sign for psychological problems. Journal (Canadian Dental Association) 2006 Mar;72(2):155-60.

32. KOYANO K, Tsukiyama Y, Ichiki R, Kuwata T. Assessment of bruxism in the clinic. Journal of oral rehabilitation 2008 Jul;35(7):495-508.

33. VANDERAS AP, Manetas KJ. Relationship between malocclusion and bruxism in children and adolescents: a review. Pediatric dentistry 1995 Jan-Feb;17(1):7-12.

34. CARIOLA TC. O desenho da figura humana de crianças com bruxismo. Bol psicol 2006 jun;56(124):37-52.

RECEBIDO EM 22/09/2017

ACEITO EM 23/10/20170 